\title{
Effect of Yi Fu Kang Oral Liquid on Gastrointestinal Side Effects Caused by Chemotherapy
}

\author{
S Hao ${ }^{1}, \mathrm{H} \mathrm{Lv}^{2}, \mathrm{X} \mathrm{Geng}^{1}, \mathrm{X} \mathrm{Wang}^{1}{ }^{1} \mathrm{X} \mathrm{Li}^{3}, \mathrm{~J} \mathrm{Shen}^{4}, \mathrm{Z} \mathrm{Zhang}^{1}$
}

\begin{abstract}
Objective: We aimed to investigate whether Yi Fu Kang oral liquid (YFK) could reduce the gastrointestinal side effects caused by chemotherapy.

Methods: We randomized 40 animals into four groups for treatment: Control $(0.8 \mathrm{~mL} 0.9 \%$ saline solution /100 g body weight/day), Ou Li shu(0.8 mL/100 g body weight/day), YFK (1 mL/100 g body weight/day) and YFK groups ( $2 \mathrm{~mL} / 100 \mathrm{~g}$ body weight/day) ( $\mathrm{n}=10$ in each group). The latency time to frst emesis and the total number of emetic behaviours were recorded. The gastrointestinal function of YFK was assessed by observing gastric remnant rate of methyl orange and charcoal powder propelling test of small intestine. Gross mucosal damage was assessed in a blind manner by calculation of a lesion index.

Results: Different dosages of Yi Fu Kang oral liquid can obviously promote the gastric emptying $(P<0.01)$ and the promotion of small intestine function $(P<0.01)$. Yi Fu Kang oral liquid can also protect the gastric mucosa and reduce the emetic response.
\end{abstract}

Conclusion: Yi Fu Kang oral liquid can enhance the function of gastrointestinal of animals by reducing the number of emetic responses, protecting the gastric mucosa and propelling movement of small intestine.

Keywords: Gastrointestinal reaction, pharmacodynamics, pigeon, rat, Yi Fu Kang oral liquid

From: ${ }^{1}$ Drug and Equipment Section, 371 Central Hospitals of People's Liberation Army, Xinxiang, 453000, Henan, China, ${ }^{2}$ Department of cardiology, 371 Central Hospital of People's Liberation Army, 453000, Henan, China, ${ }^{3}$ Graduate School of Dalian Medical University, 116044, 2013 grade, Liaoning Province, China, and ${ }^{4}$ Department of Intervention Section, 371 Central Hospital of People's Liberation Army, 453000, Henan Province, China.

Correspondence: Dr Z Zhang, 210 Wenhua Road, 371 Central Hospital of People's Liberation Army, 453000 Xinxiang, Henan Province, China. Fax +86373-3541018, e-mail: zhengc_z@163.com 


\section{INTRODUCTION}

Cancer has become one of the most important threats to human health and life all over the world. The most prevalent cancers were lung (13.0\%), breast (11.9\%) and colorectum (9.7\%). GLOBOCAN 2008 reported that there were 12.7 million new cancer cases and 7.6 million cancer deaths worldwide. It is estimated that, annual new cases and deaths of the disease will rise to 15 million and 10 million respectively in 2020 (1). Increases in cancer prevalence have been leading to the ever-improving treatment modalities. On top of surgery and radiotherapy, the use of chemotherapy as well as targeted therapy has increased along with the availability of supportive treatment for its side effect management (2). Generally, physical side effects caused by chemotherapy have been well-characterized $(3,4)$. Among the most common chemotherapy-induced side effects are bone marrow suppression, neuropathies, gastrointestinal disorders , hair loss, fatigue and skin disorders. Chemotherapy-induced nausea and vomiting are major inconveniences of chemotherapy, and may present during or soon after chemotherapy administration (5). Effective management of chemotherapy induced adverse effects is important to improve quality of life of patients which may eventually influence their willingness to complete the treatment (6).

Several Chinese herbs have been identified as potential sources of antitumor drugs (7). The effect-enhancing and toxicity-reducing activity of Chinese herbs in radiotherapy and chemotherapy of tumors was previously confirmed $(8,9)$. The symptoms of qi and yin deficiency are similar to chemotherapy- or radiotherapy-induced side effects. On the basis of the traditional Chinese medicine (TCM) consideration, Yi Fu Kang oral liquid (modified from a TCM recipe "Sheng Mai Yin") is selected as a tonic in combination with chemotherapy, for example, supplementing qi (means vital energy) and nourishing yin (means passive force) 
(10). Many studies have shown that TCM formulae exhibit more significant synergistic effects than all the ingredients combined. However, no studies focusing on the antitumor and toxicity-reducing effects of $\mathrm{Yi} F \mathrm{Fu}$ Kang oral liquid (YFK) have been reported thus far. Therefore, the present investigation was conducted to ascertain whether the YFK extract reduced toxicity of chemotherapy as well as potential mechanisms involved.

\section{MATERIALS AND METHODS}

\section{Animals and Reagents}

This work was approved by 371 Central Hospitals of People's Liberation Army. Healthy pigeons (weighing 300-450 g), Sprague Dawley rats (weighing 180-210 g) and Konmin mouse (weighing 18-22 g) were used in this study. All animals were purchased from Henan Medical University Animal Center (Zhengzhou, China) and maintained in a temperature controlled room with a $12 \mathrm{~h}$ light/dark cycle and with access to regular chow and water. All the experimental protocols were approved by the Ethics Committee of the Chinese people's liberation army 371 hospital. Ou Li shu oral liquid was purchased from Xinxiang yuanda pharmaceutical Company. Copper sulfate was purchased from Zhengzhou chemical reagent factory. Methyl orange was purchased from Shanghai chemical reagent factory.

\section{YFK preparation}

The drugs present in YFK were obtained from the Pharmacy Department of Chinese people's liberation army 371 hospital (Zhengzhou, China). The quality of the raw herbs was controlled 
according to the requirements of the Pharmacopoeia of the People's Republic of China. An aqueous extract of YFK was prepared in accordance with the following procedure. In brief, the components (as shown in Table 1) were mixed in proportion and were macerated for $1 \mathrm{~h}$ with 8 volumes of distilled water and then decocted for $40 \mathrm{~min}$. The cooled extract was filtered. The extraction procedure was repeated three times. The extracts were then combined and concentrated by boiling to a final concentration of $1.15-1.30 \mathrm{~g} / \mathrm{ml}\left(80^{\circ} \mathrm{C}\right)$. Ethanol was added to the extracts (final concentration $60 \%$ ) overnight in order to remove the polysaccharides. After recycling ethanol, adding the single syrup and adjusting PH 5.0 to 7.0, the extracts were added water to $1000 \mathrm{ml}$ and sterilized at $100^{\circ} \mathrm{C}$ for 30 minutes.

\section{Copper sulfate induced emesis}

Forty pigeons were randomly divided into four experimental groups: Control $(0.8 \mathrm{~mL} 0.9 \%$ saline solution /100 g body weight/day), Ou Li shu( $0.8 \mathrm{~mL} / 100 \mathrm{~g}$ body weight/day), YFK (1 $\mathrm{mL} / 100 \mathrm{~g}$ body weight/day) and YFK groups ( $2 \mathrm{~mL} / 100 \mathrm{~g}$ body weight/day) ( $\mathrm{n}=10 \mathrm{in}$ each group). The animals were then treated with or without drug (once a day) for 3 days. During the administration period, food and water were available ad libitum. Thirty minutes after the last administration, copper sulfate $\left(0.3 \mathrm{~g} \mathrm{~kg}^{-1}\right)$ was administered intragastrically. Each pigeon was used once. Vomiting and retching associated with and without oral expulsion, respectively, were considered as the emetic response (11). The emetic responses were characterized by a bout of emesis and there was more than an emetic behaviour within one bout. One hour after the last dose, the latency time to frst emesis, the number of bouts of emesis and the total number of emetic behaviours were recorded. 


\section{Ethanol-Induced Gastric Mucosal Damage}

We randomly divided 40 Sprague Dawley rats into four groups ( $\mathrm{n}=10$ rats per group) for treatment as described above. Rats fasted for $24 \mathrm{~h}$ before the experiment but had free access to water. After $90 \mathrm{~min}, 75 \%$ ethanol was given orally to each animal at $10 \mathrm{~mL} / \mathrm{kg}$ to induce gastric ulceration. At $4 \mathrm{~min}$ after ethanol administration, rats were killed by cervical dislocation. The stomach was removed and gross mucosal damage was assessed in a blind manner by calculation of a lesion index by use of a 0-3 scoring system based on the number and severity factor of lesions as described previously (12). The severity factor was defined according to the length of the lesions. Severity factor $0=$ no lesions visible; I=lesions $<1 \mathrm{~mm}$; II=lesions $2-4 \mathrm{~mm}$; III=lesions $>4 \mathrm{~mm}$. The lesion index was calculated as the total number of lesions multiplied by their respective severity factor.

\section{Measurement of gastric emptying}

Gastric emptying was assessed using methyl orange recovery method. The mice were randomly allocated into four groups (n=10 mice per group) for treatment as described above. Animals were deprived of food for $12 \mathrm{~h}$ with free access to tap water. Methyl orange $(0.1 \%$ $(\mathrm{wt} / \mathrm{vol}), 0.2 \mathrm{~mL})$ was given intragastrically through a stainless steel feeding tube equipped with a $1-\mathrm{mL}$ syringe to conscious rats. The mice were sacrificed $20 \mathrm{~min}$ later through cervical dislocation. The abdominal cavity was opened, the gastric cardia and pylorus were clamped, and the stomach was removed, rinsed, and placed into $10 \mathrm{~mL}$ of distilled water. The gastric contents were homogenised with $10 \mathrm{~mL}$ of $\mathrm{NaOH}$ solution $(0.5 \%$ (wt/vol)) and allowed to 
settle for $60 \mathrm{~min}$ at room temperature. Then, the mixture was centrifuged for $10 \mathrm{~min}$ (3500 rpm). The standard $0 \%$ emptying was defined by homogenisation through the above-mentioned extracorporeal operation. All of the samples were analysed by spectrophotometry at $420 \mathrm{~nm}$ to determine the absorbance values(13). Gastric emptying was calculated as residual rate of methyl orange $=$ (absorbance of test sample/absorbance of standard).

\section{Charcoal powder propelling test of small intestine(14)}

Animals were deprived of food for $12 \mathrm{~h}$ with free access to tap water. Thirty minutes after the last administration, all animals were given intragastrically charcoal powder $(10 \%(\mathrm{wt} / \mathrm{vol})$ suspended liquid $0.2 \mathrm{~mL} / 10 \mathrm{~g}$. Twenty minutes later the mice were killed by cervical dislocation. The small intestine from pylorus to the boundary of ileum and cecum was isolated and its length was"total length of small intestine". The length from pylorus to the foreland of charcoal powder was"charcoal powder propelling length". Charcoal powder propelling ratio $=[$ charcoal powder propelling length $(\mathrm{cm}) /$ total length of small intestine $(\mathrm{cm})] \times 100 \%$.

\section{Statistical Analysis.}

The software package SPSS version 17.0 (SPSS, Chicago, IL, United States) was used for statistical analyses. One-way analysis of variance and least significant difference tests were used. Results were expressed as mean \pm SD. $\mathrm{P}<0.05$ was considered to indicate a statistically significant difference. 


\section{RESULTS}

\section{Copper sulfate induced emesis in pigeons}

Intragastrically administered copper sulfate at a dose of $0.3 \mathrm{~g} \mathrm{~kg}^{-1}$ induced emesis in all of the injected pigeons with a latency of $16.4 \pm 5.2 \mathrm{~min}(\mathrm{n}=10) . \mathrm{Ou} \mathrm{Li}$ shu $(0.8 \mathrm{~mL} / 100 \mathrm{~g}$ body weight/day) markedly reduced the copper sulfate induced responses during the $1 \mathrm{~h}$ observation period. Pretreatment with YFK (2 mL/100 g body weight/day) completely suppressed the copper sulfate induced emetic responses during the $1 \mathrm{~h}$ observation period $(P<0.05)$ (Table 2).

\section{Ethanol-Induced Gastric Mucosal Damage}

This finding was similar to previous reports showing that ethanol intoxication caused extensive and acute haemorrhagic lesions on the mucosal surface of the gastric tissue and damaged gastric layers(15). As shown in Table 3 , intragastrically administered of Ou Li shu $(0.8 \mathrm{~mL} / 100 \mathrm{~g}$ body weight/day) substantially reduced gastric mucosal damage caused by subsequent challenge of the gastric mucosa with $2 \mathrm{ml}$ of $75 \%$ ethanol (lesion index $44.8 \pm 11.8$ vs $62.8 \pm 13.4$ in vehicle-treated controls, $P<0.05)$. Pretreatment with YFK groups reduced gastric mucosal damage caused by $75 \%$ ethanol in a dose-dependent manner. YFK $(1 \mathrm{~mL} / 100$ g body weight/day) reduced the lesion from $62.8 \pm 13.4$ in controls to $26.5 \pm 9.0(P<0.05)$. YFK groups ( $2 \mathrm{~mL} / 100 \mathrm{~g}$ body weight/day) conferred near-maximal protection against gastric mucosal damage caused by $75 \%$ ethanol. 


\section{Gastric emptying rate of methyl orange solution}

The amount of methyl orange recovered from the stomach was negatively correlated with the gastric empting rate. Compared with the calculated output of gastric emptying for each control animal, the YFK oral liquid rats exhibited increased values $(25 \pm 7 \%$ and $24 \pm 10 \%, \mathrm{P}<$ 0.01), whereas the control group exhibited higher residual rate of methyl orange $(42 \pm 10 \%$, Table 4).

\section{Effect of YFK on charcoal powder propelling movement of small intestine in mice}

The charcoal powder propelling ratio in normal control was $68.9 \pm 9.8 \%$, while it greatly increased to $77.0 \pm 5.8 \%$ in $\mathrm{Ou} \mathrm{Li} \mathrm{shu(}(0.8 \mathrm{~mL} / 100 \mathrm{~g}$ body weight/day) group. Comparing with control group, YFK (1, $2 \mathrm{~mL} / 100 \mathrm{~g}$ body weight/day) induced obvious increase of the charcoal powder propelling ratio of small intestine, and reached $81.9 \pm 16.2 \%$ and $82.8 \pm 7.2 \%$ $(P<0.01)$ (Table 4).

\section{DISCUSSION}

Nausea and emesis are important factors that reduce drug compliance in patients receiving anticancer chemotherapeutic (16). Due to the side effects of chemotherapy, the search for and use of complementary and alternative medicines (CAM) has increased during the past several years around the world (17). TCM is one of the most common CAMs used in China, and it has an over 5,000-year history of applications for various diseases, including gastrointestinal 
disorder (18).

YFK has long been used for the treatment of gastrointestinal disorders such as chronic gastritis and gastric and duodenal ulcer, and it could effectively attenuate nausea and vomiting. YFK could play a good supporting role in suppressing tumors and confer a protective effect on gastrointestinal mucosa damage induced by chemotherapy

Yi Fu Kang oral liquid is one of the famous Chinese medicines used to replenish qi and invigorate the functions of the spleen. It is used when there is a deficiency of qi of the spleen and stomach, which is marked by anorexia and loose bowels. Modern pharmacological studies show that this prescription can improve gastrointestinal function and strengthen immune function. Therefore, we hypothesized that this prescription may be beneficial for the restoration of intestinal function after chemotherapy.

In the current study, we used a controllable copper sulfate induced emesis rabbit pigeon model to investigate the effects of YFK on the restoration of stomach function after chemotherapy. We found that the YFK oral liquid can reduce the latency time to frst emesis, the number of bouts of emesis and the total number of emetic behaviours. YFK oral liquid treatment significantly alleviated stomach mucosal damage, promoted the gastric emptying, and enhanced the small intestine function. We observed the effect of YFK oral liquid on charcoal powder propelling movement of small intestine in mice. The result showed that YFK oral liquid increased the charcoal powder propelling ratio of small intestine, which indicates that YFK oral liquid can enhance the function of small intestinal peristalsis of mice. Furthermore, YFK oral liquid can alleviate stomach mucosal damage caused by $75 \%$ ethanol.

Although we found that the famous Chinese herbal formula YFK oral liquid has the ability to 
promote the restoration of intestinal function by regulating the intestinal homeostasis, the precise mechanisms involved are still to be elucidated. YFK oral liquid as a potential therapeutic drug for intestinal obstruction treatment needs to be investigated in the future.

\section{CONCLUSION}

The present study clearly demonstrates that YFK oral liquid promotes the restoration of intestinal function after chemotherapy by regulating intestinal homeostasis. Our results indicate that the YFK oral liquid may be a potential therapeutic drug for the side effects of chemotherapy. Further studies are needed to assess the side effects and limitations of this drug in the treatment of gastrointestinal diseases.

\section{Acknowledgments}

No funding and financial disclosure

\section{Conflict of Interests}

The authors declare that there is no conflict of interests regarding the publication of this paper. 


\section{REFERENCES}

1. Jemal A, Bray F, Center MM, Ferlay J, Ward E, Forman D. Global cancer statistics. CA Cancer J Clin 2011; 61: p. 69-90.

2. Dohler N, Krolop L, Ringsdorf S, Meier K, Ko YD, Kuhn W, et al.Task allocation in cancer medication management - integrating the pharmacist. Patient Educ Couns 2011; 83: p. $367-74$.

3. Arslan FT, Basbakkal Z, Kantar M. Quality of life and chemotherapy-related symptoms of Turkish cancer children undergoing chemotherapy. Asian Pac J Cancer Prev 2013; 14: p. 1761-8.

4. Chan HK,Ismail S. Side Effects of Chemotherapy among Cancer Patients in a Malaysian General Hospital: Experiences, Perceptions and Informational Needs from Clinical Pharmacists. Asian Pac J Cancer Prev 2014; 15: p. 5305-9.

5. Faria C, Li X, Nagl N, McBride A Outcomes Associated with 5-HT3-RA Therapy Selection in Patients with Chemotherapy-Induced Nausea and Vomiting: A Retrospective Claims Analysis. Am Health Drug Benefits 2014; 7: p. 50-8.

6. Goyal RK, Wheeler SB, Kohler RE, Lich KH, Lin CC, Reeder-Hayes K. Health care utilization from chemotherapy-related adverse events among low-income breast cancer patients: effect of enrollment in a medical home program. N C Med J 2014; 75 : p. 231-8.

7. Wang CY, Bai XY, Wang CH. Traditional Chinese medicine: a treasured natural resource of anticancer drug research and development. Am J Chin Med 2014; 42: p. 543-59. 
8. Zhang $\mathrm{HB}$, Lu P, Cao WB, Zhang ZH, Meng XL. The effect-enhancing and toxicity-reducing activity of Thunb. extract in murine liver cancer chemotherapy. Mol Clin Oncol 2013; 1(2): p. 395-399.

9. Zhu WR, Zheng L, Guo YB, Yuan JM, Shen XH. Clinical research of intraperitoneal chemotherapy plus Shenmai Injection in treating advanced colorectal cancer. Zhong Xi Yi Jie He Xue Bao 2005; 3: p. 266-9.

10. Chen Z, Wang P, Huang WX, Liu LM. Experimental study on effects of shengmai injection: enhancing 5-FU anti-tumor efficacy and reducing its toxicity. Zhong Xi Yi Jie He Xue Bao 2005;3: p. 476-9.

11. Tanihata S, Oda S, Nakai S, Uchiyama T. Antiemetic effect of dexamethasone on cisplatin-induced early and delayed emesis in the pigeon. Eur J Pharmacol 2004; 484: p. 311-21.

12. Stroff T, Plate S, Ebrahim JS, Ehrlich KH, Respondek M, Peskar BM. Tachykinin-induced increase in gastric mucosal resistance: role of primary afferent neurons, CGRP, and NO. Am J Physiol 1996; 271: p. G1017-27.

13. Tian ZB, Deng RJ, Sun GR, Wei LZ, Kong XJ, Ding XL, et al. Expression of gastrointestinal nesfatin-1 and gastric emptying in ventromedial hypothalamic nucleus- and ventrolateral hypothalamic nucleus-lesioned rats. World J Gastroenterol 2014; 20: p. 6897-905.

14. Zhang HQ, Zhou CH, Wu YQ. Effect of emodin on small intestinal peristalsis of mice and relevant mechanism. World J Gastroenterol 2005;11: p. 3147-50.

15. Alrashdi AS, Salama SM, Alkiyumi SS, Abdulla MA, Hadi AH, Abdelwahab SI, et al. 
Mechanisms of Gastroprotective Effects of Ethanolic Leaf Extract of Jasminum sambac against $\mathrm{HCl} /$ Ethanol-Induced Gastric Mucosal Injury in Rats. Evid Based Complement Alternat Med2012; 786426: p. 5.

16. Van den Brande J, Brouwer A, Peeters M. Use of antiemetics in the prevention of chemotherapy-induced nausea and vomiting: review and focus on the Belgian situation. Acta Gastroenterol Belg 2014; 77: p. 240-8.

17. Weeks L, Balneaves LG, Paterson C, Verhoef M. Decision-making about complementary and alternative medicine by cancer patients: integrative literature review. Open Med 2014;8:e54-e66. eCollection 2014.

18. Chen KC, Lee WY, Chen HY, Chen CY. In Silico Investigation of Potential TRAF6 Inhibitor from Traditional Chinese Medicine against Cancers. Biomed Res Int 2014; 429486: p. 25. 
Herb Effect on Side Effects by Cheemotherapy

Table 1 Standard formulation of Yi Fu Kang oral liquid (YFK)

\begin{tabular}{ll}
\hline Pinyin name & Latin name \\
\hline (1) Renshen & Panax ginseng C.A.Mey. \\
(2) Shayuanzi & Astragalus complanatus R.Br. \\
(3) Dangggui & Angelica sinensis (Oliv.)Diels \\
(4) Chuanshanjia & Manis pentadactyla Linnaeus \\
(5) Xuanshen & Scrophularia ningpoensis Hemsl. \\
(6) Maidong & Ophiopogon japonicas (L.f) Ker Gawl. \\
\hline
\end{tabular}

Table 2 Effect of YFK oral liquid on Copper sulfate induced emesis in pigeons

\begin{tabular}{llll}
\hline Groups & Dose $(\mathrm{mL} / 100 \mathrm{~g})$ & Latency $(\mathrm{min})$ & $\begin{array}{l}\text { Total number of } \\
\text { emetics }(\text { Times } / \mathrm{h})\end{array}$ \\
\hline Control & 0.8 & $16.4 \pm 5.2$ & $54.7 \pm 28.9$ \\
Ou Li shu & 0.8 & $18.0 \pm 6.9$ & $27.4 \pm 14.4^{+}$ \\
YFK & 1 & $19.5 \pm 8.1$ & $30.8 \pm 14.7^{+}$ \\
YFK & 2 & $26.2 \pm 10.5^{+}$ & $22.3 \pm 13.0^{++}$ \\
\hline
\end{tabular}

Values are mean $\pm \mathrm{SD}(n=10)$. Differs from Control, ${ }^{+} P<0.05,{ }^{++} P<0.01$. 
Table 3 Effect of YFK oral liquid on ethanol-induced gastric mucosal damage

\begin{tabular}{llll}
\hline Groups & Dose $(\mathrm{mL} / 100 \mathrm{~g})$ & Lesion index & Inhibition ratio $(\%)$ \\
\hline Control & 0.8 & $62.8 \pm 13.4$ & \\
Ou Li shu & 0.8 & $44.8 \pm 11.8$ & 28.7 \\
YFK & 1 & $39.6 \pm 11.5$ & 36.9 \\
YFK & 2 & $26.5 \pm 9.0$ & 57.8 \\
\hline
\end{tabular}

Values are mean $\pm \mathrm{SD}(n=10)$. Differs from Control, ${ }^{+} P<0.05,{ }^{++} P<0.01$.

Table 4 Effect of YFK oral liquid on gastric emptying and propelling test of small intestine

\begin{tabular}{llll}
\hline Groups & Dose $(\mathrm{mL} / 100 \mathrm{~g})$ & $\begin{array}{l}\text { Residual rate of } \\
\text { methyl orange }(\%)\end{array}$ & $\begin{array}{l}\text { Charcoal powder } \\
\text { propelling ratio }(\%)\end{array}$ \\
\hline Control & 0.8 & $42 \pm 10$ & $68.9 \pm 9.8$ \\
Ou Li shu & 0.8 & $28 \pm 8^{++}$ & $77.0 \pm 5.8$ \\
YFK & 1 & $25 \pm 7^{++}$ & $81.9 \pm 16.2^{++}$ \\
YFK & 2 & $24 \pm 10^{++}$ & $82.8 \pm 7.2^{++}$ \\
\hline
\end{tabular}

Values are mean $\pm \mathrm{SD}(n=10)$. Differs from Control, ${ }^{+} P<0.05,{ }^{++} P<0.01$. 